\title{
Performance evaluation of hierarchical clustering protocols with fuzzy C-means
}

\author{
Hamid Barkouk, EL Mokhtar En-Naimi, Aziz Mahboub \\ LIST Laboratory, Department of Computer Sciences, Faculty of Sciences and Technologies, Abdelmalek Essaadi \\ University, Morocco
}

\begin{tabular}{l} 
Article Info \\
\hline Article history: \\
Received Sep 7, 2020 \\
Revised Dec 19, 2020 \\
Accepted Jan 13, 2021 \\
\hline
\end{tabular}

\section{Keywords:}

Energy consumption Energy efficiency

Fuzzy means Network lifetime Routing protocols Subtractive clustering method

\begin{abstract}
The longevity of the network and the lack of resources are the main problems within the WSN. Minimizing energy dissipation and optimizing the lifespan of the WSN network are real challenges in the design of WSN routing protocols. Load balanced clustering increases the reliability of the system and enhances coordination between different nodes within the network. WSN is one of the main technologies dedicated to the detection, sensing, and monitoring of physical phenomena of the environment. For illustration, detection, and measurement of vibration, pressure, temperature, and sound. The WSN can be integrated into many domains, like street parking systems, smart roads, and industrial. This paper examines the efficiency of our two proposed clustering algorithms: Fuzzy C-means based hierarchical routing approach for homogeneous WSN (F-LEACH) and fuzzy distributed energy efficient clustering algorithm (F-DEEC) through a detailed comparison of WSN performance parameters such as the instability and stability duration, lifetime of the network, number of cluster heads per round and the number of alive nodes. The fuzzy C-means based on hierarchical routing approach is based on fuzzy C-means and low-energy adaptive clustering hierarchy $(\mathrm{LEACH})$ protocol. The fuzzy distributed energy efficient clustering algorithm is based on fuzzy C-means and design of a distributed energy efficient clustering (DEEC) protocol. The technical capability of each protocol is measured according to the studied parameters.
\end{abstract}

This is an open access article under the CC BY-SA license.

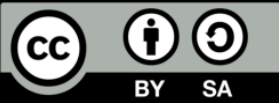

\section{Corresponding Author:}

\section{Aziz Mahboub}

LIST Laboratory, Department of Computer Sciences

Faculty of Sciences and Technologies, Abdelmalek Essaadi University

B.P 410, Route de Charf, Tangier, Morocco

Phone : +212666145279

Email: amahboub@uae.ac.ma

\section{INTRODUCTION}

WSN is one of the main technologies devoted to the identification, sensing, and controlling of physical environmental phenomena in real-time such as the detection and measurement of vibration, pressure, temperature and sound. [1-3]. The WSN can be incorporated in many sectors like street parking, smart roads, and industrial monitoring [4-5]. In general, WSN consists of several self-organized sensor nodes with limited energy, computational capacities, and bandwidth [6-8], which are deployed inarge quantitiesof sensorsin dedicated environments. Under hostile conditions, adjusting the node's battery under operating mode is impractical. An autonomous energy source (battery) supplies each sensor node and is fitted with a microcontroller, memory, and transceiver [2-7]. The base station (Sink), however, others data for processing and sends it to the central computer. A WSN managed by one or more base stations $[1-4,9]$. 
In WSN the communication process requires a considerable quantity of energy. Energy consumptin optimization is one of the prominent problems facing wireless sensor networks. For this reason, improving the network's energy output is necessary in order to increase the system's lifetime [9]. To overcome this limitation a set of techniques have been developed to minimize the communication energy consumption [5]. Due to their energy efficiency, automated routing strategies such as single-hop,multi hop or clustering have been widely implemented at WSN [6].

Several researchers have proposed the routing algorithms to increase the lifetime of network. Based on the network layout, routing in WSN can typically be classified into flat routing, hierarchical routing, and location routing [10-12]. Among such protocols are the routing protocols for clustering. The network is partitioned into small areas in the clustering algorithm, and each part is controlled and regulated by the cluster head $(\mathrm{CH})$. This is responsible for compressing the collected data and transmitting it to the base station through a single hop or multi-hop that can be linked to a powerful device over the internet or a satellite of the information sensed by the area nodes [12-16]. The clustering protocols saves resources to extend the network's lifespan [16]. That can also be a realistic attribute for large sensor networks, since the cluster heads are easier to handle than the whole network. Multiple clustering routing protocols using multi-paths have been proposed on the basis of load balancing according to the following order LEACH [16-19], DEEC [12, 15, 16]. The clustering protocols can be divided into two types: the clustering algorithm with homogeneous schemes in which all the sensor nodes have the same initial energy; and the heterogeneous clustering protocols in which all the sensor nodes are delivered with a different amount of power at network startup [18].

In this paper, we conduct an analysis of the performance parameters of some effective hierarchical routing protocols, the low-energy adaptive hierarchy protocol known as LEACH [6], the stable election protocol (SEP) [20], the distributed energy-efficient clustering protocol (DEEC) [12], and our porpositions: the F-LEACH protocol and the F-DEEC protocol. The rest of the paper is structured as follows: The relevant research and benchmarking are presented in section 2. Section 3 introduces introduces the F-LEACH protocol. Section 4 describes the F-DEEC protocol. Section 5 establishes models and the analysis of the results. Eventually, section 6 finalizes the paper based on the findings obtained and possible research

\section{RELATED WORK AND BENCHMARKING}

In the recent past, a number of clustering algorithms were proposed for WSNs, among these are proposed found towards effective data communication and data processing with optimal resource usage in the WSN. In this part, we describe some of the most effective routing algorithms of WSN. Researchers have conducted a variety of works focused on application and network layout to advance routing protocols in WSN. There are also several parameters to consider when designing the routing protocol. Energy performance is one of the most obvious variables directly impacting the lifespan of the network. In this paper, we provide some examples of the energy-efficient routing protocols.

\subsection{Low-energy adaptive clustering hierarchy (LEACH)}

The LEACH protocol $[8,18]$ is the first protocol that uses a pure probabilistic model to pick CHs and to rotate the $\mathrm{CHs}$ periodically to balance energy usage. The dynamic clustering mechanism has been introduced, where a node elects itself by unique probability to become a $\mathrm{CH}$ and transmits its status to all nodes $[3,21]$. In certain instances, however, inefficient $\mathrm{CHs}$ are selectable. This is due to the fact that LEACH only relies on a probabilistic model. Some CHs may be very close to each other and maybe situated at the WSN's edge. Such inefficient heads of Clusters can have a negative impact on energy efficiency in the network. The LEACH cycle moves to circles. Every round has two phases: the first phase is set- up for organizing the clusters and selecting $\mathrm{CHs}$; the second phase is stable-state for transmitting data to the base station. $\mathrm{CHs}$ elections are based on the desired percentage of $\mathrm{CHs}$ and the number of iterations a node has taken on CHs function [22, 23]. A node $s$ is therefore a random value between 0 and 1 . If the value is below the T(s) threshold, the node will become $\mathrm{CH}$. The threshold is set as:

$$
T(s)=\left\{\begin{array}{cc}
\frac{p}{1-p\left(\operatorname{rmod}\left(\frac{1}{p}\right)\right)} & \text { if } s \in G \\
0 & \text { otherwise }
\end{array}\right\}
$$

where $p$ is the target percentage of $\mathrm{CH}$ nodes within the sensor population and $\boldsymbol{r}$ is the current round number, where $\mathrm{G}$ is the set of nodes that were not $\mathrm{CHs}$ in the last $1 / p$ rounds $[3,12,24]$. With all the advantages of the $\mathrm{LEACH}$ protocol, by dispersing the cluster heads across the network, it suffers from the development of quality clusters. Therefore, the authors in $[13,19]$ proposed LEACH-C recommend a centralized approach to selecting $\mathrm{CHs}$ for an improved version of LEACH. In LEACH-C all nodes are sent to the base station with their id, 
energy level, and GPS coordinates. It is the base station's role to select a few nodes as $\mathrm{CH}$ based on their energy level. For the current round, nodes with energy, greater than average energy, are selected as $\mathrm{CH}$. The major downside of this approach is that it dissipates the energy of all nodes in each round for the transmission of the information to BS.

\subsection{Stable election protocol (SEP)}

It makes LEACH interesting to experiment with the use of other probability-based models such as A stable election protocol (SEP) [20] as a method. The SEP protocol adopted the similar procedures adopted by the LEACH protocol for the selection of cluster heads. The choice process of cluster heads is based on the weighted probability of each node being a cluster head [7]. The SEP protocol takes into account the heterogeneities in each sensor node in the initial energy quantity. The SEP protocol categorizes the sensor nodes into two sets according to the initial energy: advanced nodes and regular nodes. Advanced nodes have initial energy that is very high than regular nodes, the additional energy factor between advanced and standard nodes is denoted by $\alpha$. The advanced nodes are equipped with $(1+\alpha)$ more energy quantity than the regular nodes. The advanced nodes in SEP protocol have more probability of being cluster head than regular nodes. The SEP uses two weighted probabilities of election: One for regular nodes, and the other for advanced nodes [13]. Where $P_{\text {normal }}$ is the weighted probability of election for normal nodes, and the $P_{a d v}$ is the weighted probability of election for the advanced nodes. Accordingly, the weighted probabilities of the usual node and advanced node are generated accordingly $[12,25]$.

$$
\begin{aligned}
& P_{\text {normal }}=\frac{p}{1+m \alpha} \\
& P_{a d v}=\frac{p}{1+m \alpha} *(1+\alpha)
\end{aligned}
$$

Where $m$ is the proportion of advanced nodes with nodes with $\alpha$ times more energy than the normal nodes.

In SEP protocol, each node type has a threshold; $T($ snormal $)$ is the normal node threshold and $T_{(\text {Sadv })}$ is the advanced node threshold. Consequently, the normal and advanced threshold for equation of nodes is:

- For normal nodes:

$$
\mathrm{T}(\text { snormal })=\left\{\begin{array}{cc}
\frac{\mathrm{P}_{\text {normal }}}{1-\mathrm{P}_{\text {normal }}\left(\operatorname{rmod}\left(\frac{1}{\mathrm{P}_{\text {normal }}}\right)\right)} & \text { ifs } \in \mathrm{G} \prime \\
0 & \text { otherwise }
\end{array}\right\}
$$

where $G^{\prime}$ is a set of normal nodes which can become $\mathrm{CH}$ and $\mathrm{m}$ is the proportion of advanced nodes with $\alpha$ times more energy than the normal nodes [26].

- For advanced nodes:

$$
\mathrm{T}(\mathrm{sadv})=\left\{\begin{array}{cc}
\frac{\mathrm{P}_{\mathrm{adv}}}{1-\mathrm{P}_{\mathrm{adv}}\left(\operatorname{rmod}\left(\frac{1}{\mathrm{P}_{\mathrm{adv}}}\right)\right)} & \text { ifs } \in \mathrm{G} \prime \prime \\
0 & \text { otherwise }
\end{array}\right\}
$$

where $G^{\prime \prime}$ is a set of advanced nodes that have not become cluster heads within the last $\frac{1}{P_{\operatorname{axdv}}}$ round.

\subsection{The distributed energy efficient clustering protocol (DEEC)}

The DEEC protocol is an energy-intensive, distributed clustering protocol for the heterogeneous wireless sensor network. Unlike the LEACH protocol and the SEP protocol, the DEEC protocol adopts an enhanced method for the collection of CHs used by the LEACH protocol and the SEP protocol, to measure the probability of the original and residual energy level of the nodes [8, 27]. The CHs are chosen by a probability on the basis of the ratio of each node's remaining energy to the average network energy. For each node, the round number of the revolving epoch is different depending on its original and residual energy. The DEEC protocol adapts each node's rotating epoch to its capacity. Nodes carrying high initial and remaining energy have greater chances of being $\mathrm{CH}$ than low-energy nodes [23, 28]. This method allows for prolonging the network lifetime of the DEEC protocol. But it is difficult to provide a global knowledge of the average energy of each node's network. According to (7), the DEEC protocol assumes an ideal value for the network lifetime used to measure the reference energy that each node will extend during each round. The DEEC has the drawback that the advanced nodes are still penalized when the residual energy is limited and when it is equal 
to regular nodes. The advanced nodes would die fast on these circumstances than the other nodes. The average probability of $\mathrm{i}^{\text {th }}$ node to be a $\mathrm{CH}$ during the next round is given by pi.

$$
p i=p \frac{E(r)}{E(r)_{i}}
$$

Where $\mathrm{p}$ is the optimal propobilty and $\mathrm{E}(\mathrm{r})$ is the average energy round, set (7).

$$
E(r)=E_{\text {total }}(1-R) / N
$$

Where $\mathrm{R}$ denotes the total rounds of the network lifetime.

The value of the total Energy is given (8).

$$
E_{\text {total }}=N *(1-m) * E o+N * m * E o *(1+\alpha)
$$

Where $R$ denotes the total rounds of the network lifetime. Let $E_{\text {round }}$ denote the energy consumed by the network in each round. $R$ can be approximated (9).

$$
R=\frac{E_{\text {total }}}{E_{\text {round }}}
$$

\subsection{Fuzzy C-means clustering algorithm}

The fuzzy C-means clustering algorithm (FCM), which was developed by Dunn in 1974 and improved by Bezdek in 1987, has been extensively studied and applied [29, 30]. FCM is a clustering algorithm that is unsupervised as same as the k-means algorithm with the same cluster division purpose. Nonetheless, $\mathrm{k}$-means is a hard set based algorithm, and FCM is an algorithm based on the non-crisps approach (all individuals are listed in two groups: 1 or 0) [30-32]. This algorithm works by assigning affiliation to each sensor node that corresponds to each cluster center. This process is based on the distance between the cluster center and the sensor node. Therefore, the closer the sensor node is to the cluster center, the stronger its membership in cluster center is [30-33]. The FCM algorithm represents an iterative optimization algorithm that minimizes the following objective function [33].

$$
f=\sum_{i=1}^{n} \sum_{j=1}^{C} u_{i j}^{m}\left\|x_{i}-C H_{j}\right\|^{2}
$$

Where $n$ is the number of sensor nodes, $c$ is the number of clusters, $x_{i}$ is the ith sensor node, $C H_{j}$ is the $\mathrm{j}^{\text {th }}$ cluster center, $\mathrm{u}_{\mathrm{ij}}^{\mathrm{m}}$ is the degree of membership of the $\mathrm{i}^{\text {th }}$ sensor node in the $\mathrm{j}^{\text {th }}$ cluster, and $m$ is a constant greater than 1 (typically $\mathrm{m}=2$ ). $\left\|\mathrm{x}_{\mathrm{i}}-\mathrm{CH}_{\mathrm{j}}\right\|^{2}$ represents the measure of the Euclidean distance between the sensor node $\mathrm{x}_{\mathrm{i}}$ and the cluster center $\mathrm{CH}_{\mathrm{j}}$.

The degree of membership $\mathrm{u}_{\mathrm{ij}}^{\mathrm{m}}$ and the cluster Head $\mathrm{CH}_{j}$ are defined as the (11), (12).

$$
\begin{aligned}
& u_{i j}=\frac{1}{\sum_{k=1}^{c}\left(\frac{\left\|x_{i}-c_{j}\right\|}{\left\|x_{i}-c_{k}\right\|}\right)^{\frac{2}{m-1}}} \\
& C H_{j}=\frac{\sum_{i=1}^{N} u_{i j}^{m} \cdot x_{i}}{\sum_{i=1}^{N} u_{i j}^{m}}
\end{aligned}
$$

Following are the steps of the fuzzy C-means algorithm

a. Initialize membership $u_{i j}$;

b. Find the fuzzy centroid $C H_{j}$ for $\mathrm{j}=\{1,2,3 \ldots \mathrm{c}\}$;

c. Update the fuzzy membership $u_{i j}$;

d. Repeat steps II and III until $f\left(u_{i j}, C H_{j}\right)$ is no longer decreasing.

\section{FUZZY C-MEANS BASED HIERARCHICAL ROUTING APPROACH (F-LEACH)}

Here we present the outline of a fuzzy C-means based hierarchical routing approach (F-LEACH). The sensor nodes are uniformly spread over an area of $100 \mathrm{~m} 2$ to track the environment incessantly. Sensor node sensing data is forwarded to BS outside of the deployment area. Each sensor node may either work in sensing 
mode to track the parameters of the environment and transmit it to the associated $\mathrm{CH}$ or in $\mathrm{CH}$ mode to collect, compress, and send data to the base station. Here follow some additional as assumptions:

- The base station has limitless power and computing power and is located outside the sensor area.

- The network is homogeneous and all sensor nodes are static and have the same initial capacity.

- Nodes have the ability to monitor the transmission power in relation to the distance of receiving nodes.

- Links are symmetric

The F-LEACH use three algorithms. Firstly, it starts using the subtractive clustering approach to evaluate the correct number of clusters [32]. The FCM algorithm is then implemented to form highly uniform clustering dispersion of nodes (segmentaion of network). In each cluster, the LEACH protocol is used to build the sub-clusters and choose the head sub-clusters and their members, which apply a pure probabilistic model for $\mathrm{CHs}$ selection and periodically rotate the $\mathrm{CHs}$ for the energy consumption balance. Figure 1 presents a flowchart of the F-LEACH cluster formation process, which combines the clustering of fuzzy C-means, LEACH, and the subtractive clustering method [33].

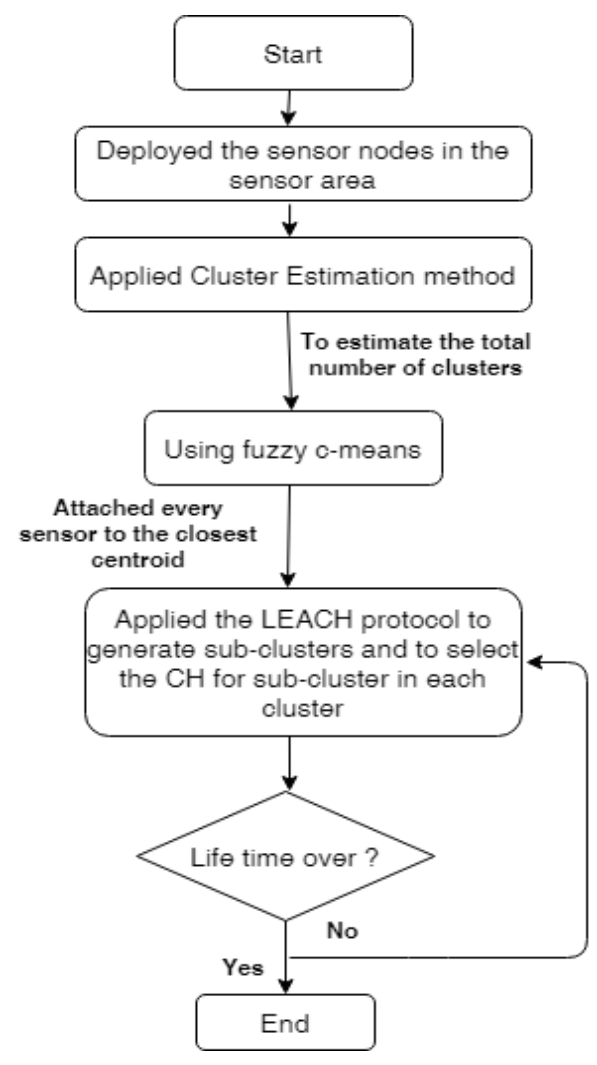

Figure 1. Flowchart of the cluster formation process of the F-LEACH

\section{DISTRIBUTED ENERGY EFFICIENT CLUSTERING ALGORITHM BASED ON FUZZY LOGIC APPROACH (F-DEEC)}

In this section, we show the distributed energy efficient clustering algorithm system based on a fuzzy logic approach (F-DEEC), which focuses on three approaches to deal with the WSN energy conservation issue [26]. The sensor nodes are randomly distributed in the application area so as to monitor the environment incessantly. Here follow some additional as assumptions: The base station has limitless power and computing power. All sensor nodes are static and have the same initial capacity. Nodes have the ability to monitor the transmission power in relation to the distance of receiving nodes. The F-DEEC model is based on three algorithms. Firstly, it starts to use the subtractive clustering approach for an optimal number of wide clusters. Secondly, the role of the application of the FCM algorithm is to form highly uniform clustering of nodes based on an optimal number generated by the subtractive clustering method. In each cluster, the DEEC protocol is used for the construction of the clusters and to the selection of the CHs and their members. To balance energy consumption, it uses a pure probabilistic model to select $\mathrm{CHs}$ and to rotate the $\mathrm{CHs}$ periodically. Figure 2 demonstrates the flowchart of the F-DEEC cluster formation process [28]. 


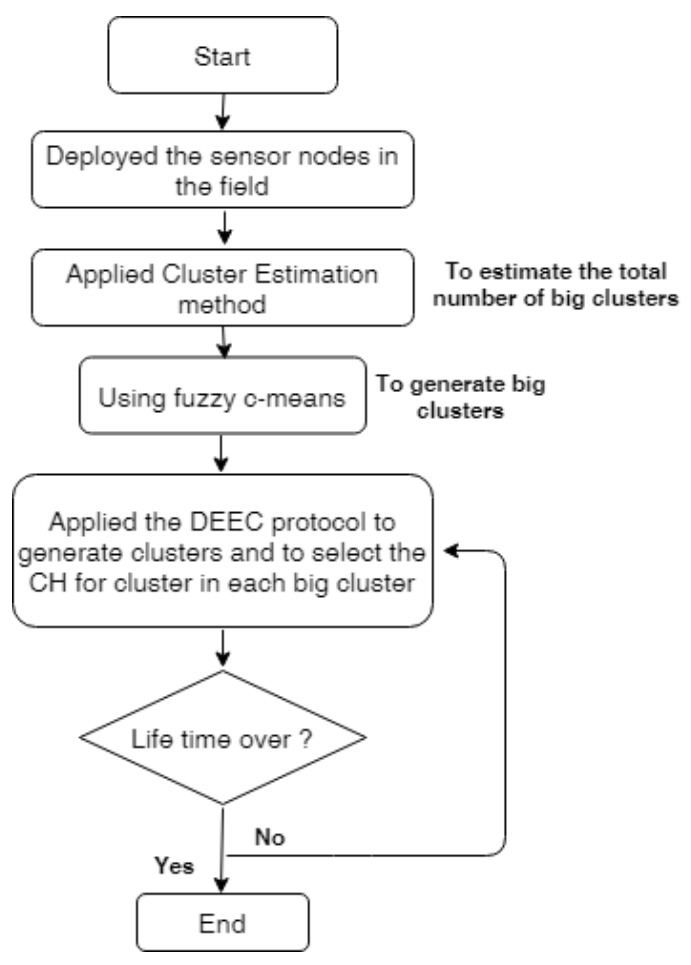

Figure 2. Flowchart of the cluster formation process of the F-DEEC

\section{SIMULATION AND EVALUATION}

To assess the F-LEACH and F-DEEC energy efficiency, we compared the main performance of the F-LEACH and F-DEEC with the DEEC protocol and the LEACH protocol. All simulations were carried out using MATLAB to evaluate the entire energy system and the number of nodes alive. We began by explaining the metrics used in the simulations. We include the details of the algorithm used, and then present and analyze all the results obtained. For simulation, 100 nodes are assumed to be distributed randomly in an area of $(100 / 100) \mathrm{m}$; the BS is positioned outside the area at the coordinate $(-50,50)$. The nodes do not have the same initial capacity, and it is presumed that the base station has limitless power. Throughout each round, each node sends.4000-bit packets to the base station via the head of the cluster.

All the results are displayed in Figures 3-7. Figure 3 shows the number of dead nodes versus the transmission series. The blue-colored curve shows the results obtained by using the LEACH protocol. The results of the DEEC protocol show the red-colored curve; the yellow-colored curve shows the results of F-LEACH and the green color shows the results of F-DEEC protocol. Figure 4 shows the number of live nodes versus the transmission round. This figure has 4 curves: The red curve shows the results obtained in the case where the DEEC protocol is executed; the results of the LEACH protocol shown by the blue curve. The yellow curve shows the results obtained by F-LEACH and the green color shows F-DEEC protocol.

According to Figures 3 and 4, we can observe that protocol prolongs the stability period compared to F-LEACH, DEEC and LEACH protocol. In addition to that, the F-LEACH gives better results in stability period than the DEEC and LEACH. According to Figures 3 and 4, we can note that F-DEEC protocol extends the stability duration compared to protocol F-LEACH, DEEC and LEACH. Furthermore, the F-LEACH provides better results in stability cycle than the DEEC and LEACH. The first dead node for LEACH protocol, DEEC protocol, F-LEACH protocol, and F-DEEC protocol is at 828 rounds, 962 rounds, 1630 rounds, 1896 rounds respectively. Statistics show that the F-LEACH protocol and the F-DEEC protocol are better than the LEACH protocol and DEEC protocol to increase the parameter stability duration. The last dead node to LEACH protocol is at 2720 rounds. For DEEC protocol, one node is live at 4000 rounds, 24 living nodes, 53 living nodes for DEEC protocol, respectively F-LEACH protocol and F-DEEC protocol. Therefore, in the instability period, the F-DEEC protocol proves to be more energy-efficient than the F-LEACH protocol, the DEEC protocol and the LEACH protocol.

With respect to the number of rounds as seen in Figure 4, we have estimated the network lifetime in terms of a number of living nodes. The alive nodes are those with their resources as not null. In the original LEACH protocol, all nodes in the network are dead for the 2720th round. But for the DEEC protocol one node is alive after the 4000th round. In the F-LEACH protocol, the first nodes die in 1630th rounds and after 4000th 
rounds the 24 nodes are alive. The first nodes in the F-DEEC protocol die in rounds 1896 and the 53 nodes are alive after rounds 4000. Among all these, the F-DEEC protocol offers the longest lifetime of the network that allows a balanced network of loads to be reached. The results obtained in Figures 4 and 5 indicated that the FDEEC protocol and the F-LEACH protocol supported WSN with the longest lifetime compared to the LEACH protocol and DEEC protocol. It can be observed that protocol F-DEEC and protocol F-LEACH increase the stability duration relative to protocol LEACH and protocol DEEC. The F-DEEC protocol and the F-LEACH protocol prove therefore to be more energy-efficient than the DEEC protocol and the LEACH protocol.

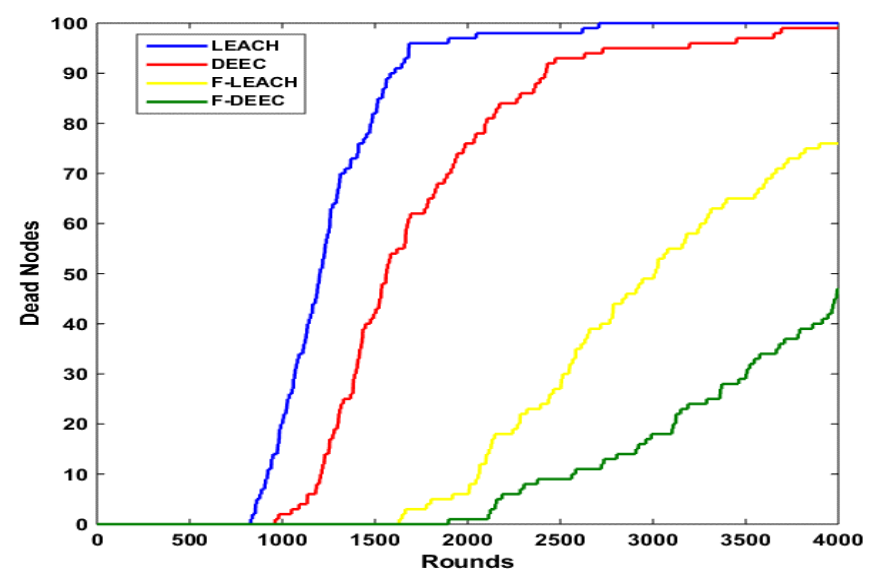

Figure 3. Number of dead nodes versus transmission round

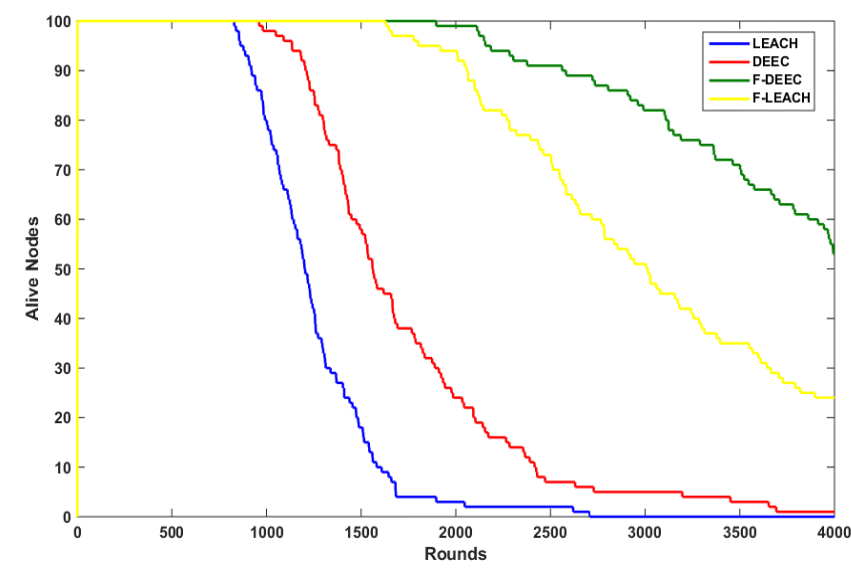

Figure 4. Number of alive nodes versus transmission round

The energy consumption for each transmission round is demonstrated in Figures 5 and 6. In Figure 5, there are 3 curves; the LECH protocol presented by the curve in blue; the red color portrays the DEEC protocol and the curve in yellow shows the result of F-LEACH protocol. Findings for F-DEEC protocol, LEACH protocol, and DEEC protocol are presented in Figure 6. In the case where F-DEEC is implemented, the green curve shows the energy consumption for each point. As for the other curves (the red curve and the blue curve), they have the same behavior of consumption of transmission energy per round.

According to Figures 5 and 6, the first protocol that absorbs more energy than the other protocols during the stability period is LEACH protocol. However, the DEEC protocol consumes less than the LEACH protocol, but more than the F-LEACH protocol and the F-DEEC protocol, while the F-DEEC protocol consumes less than the other three. We have measured the total network energy consumption per round as seen in Figure 7. The blue color indicates the LEACH protocol, the red color represents the DEEC protocol, the yellow color illustrates the F- LEACH protocol, and the green color shows F-DEEC protocol. This quantity refers to the direct volume of energy exhausted in the network per round. Here, the overall energy consumption by the network is $78.48 \mathrm{~J}, 75.38 \mathrm{~J}, 71.19 \mathrm{~J}$, and $63.93 \mathrm{~J}$ for LEACH protocol, DEEC protocol, F-LEACH 
protocol and F-DEEC protocol respectively. The performance of the F-DEEC protocol proves to be better than the LEACH protocol, the DEEC protocol, and the F-LEACH protocol. Yet, the F-LEACH protocol expended its energy more than DEEC protocol and LEACH protocol. We can see that F-DEEC protocol decreases the energy consumption by round and increases the network lifetime more than the standard DEEC protocol, the original LEACH, and F-LEACH protocol. Also, the F-LEACH protocol improved the network's lifetime compared to the LEACH and DEEC.

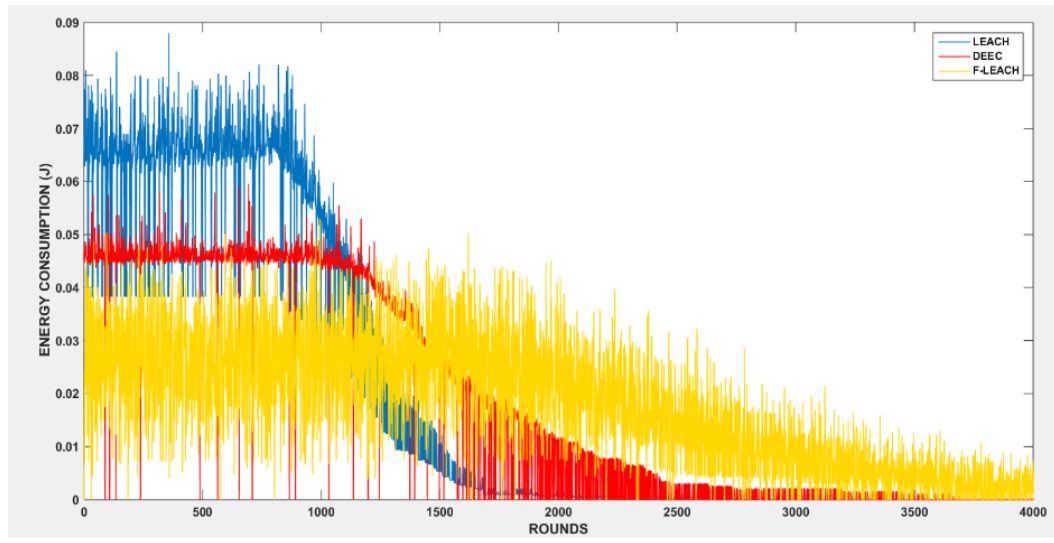

Figure 5. Energy consumption for each transmission round

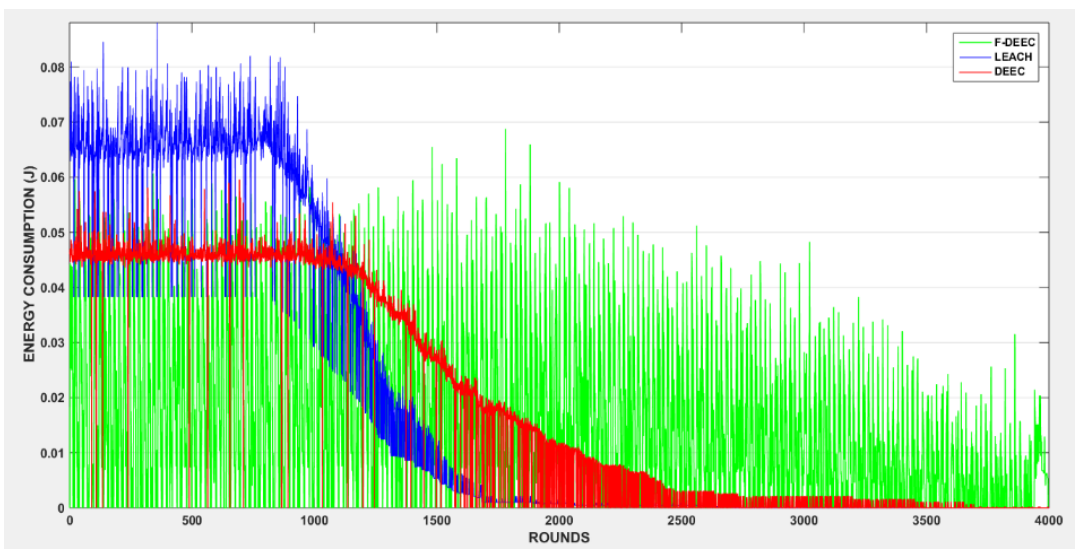

Figure 6. Energy consumption for each transmission round

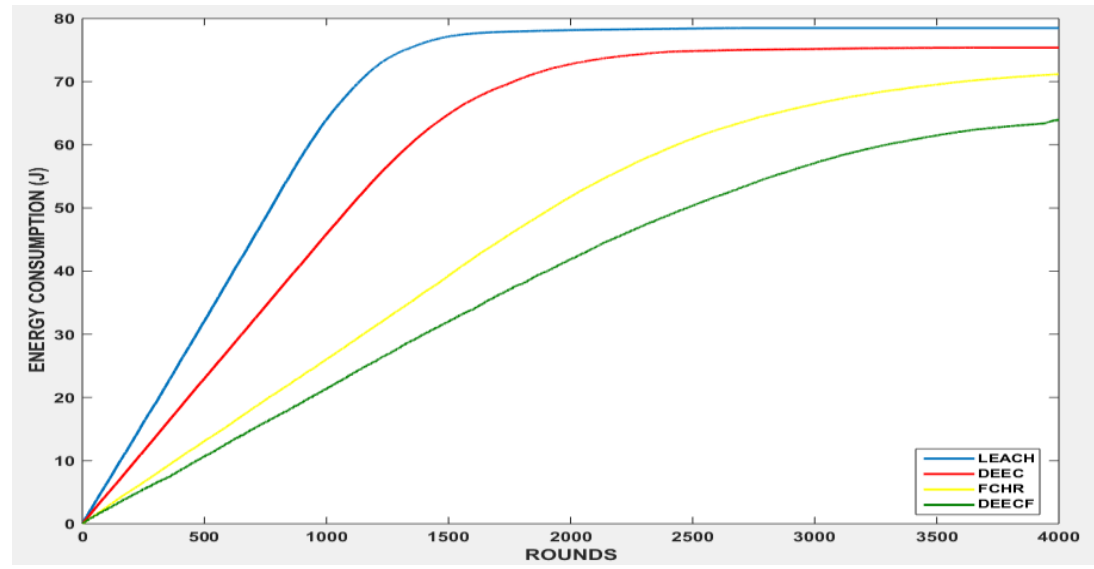

Figure 7. Total energy consumed by the network versus transmission round 


\section{CONCLUSION}

The main objective of this work is to propose tow Hybrid routing protocols based on fuzzy C-means algorithm applied for wireless sensor networks. The proposed routing protocols minimizes the energy consumption, extends the network lifetime of the sensor nodes. Through a comparison of key performance parameters of wireless sensor networks, a solid evaluation of LEACH, DEEC, F-LEACH, and F-DEEC protocols. In this respect, Extensive simulations have been carried out to assess the technical capability of each protocol in relation to the studied parameters. Therefore, the results show that the F-DEEC protocol has proven that its energy-efficiency, reliability and load balancing in the stability and instability periods of the network.

\section{REFERENCES}

[1] Noureddine, Seddiki, Khelifa, Benahmed, Mohammed, Belgachi, "Approach to minimizing consumption of energy in wireless sensor networks," International Journal of Electrical and Computer Engineering (IJECE), vol. 10, no. 3, pp. 2551-2561, 2020.

[2] Ikram D., Abdennaceur B., Abdelhakim B., "An enhanced energy-efficient routing protocol for wireless sensor network," International Journal of Electrical \& Computer Engineering (IJECE), vol. 10, no. 5, pp. 5462-5469, 2020.

[3] Kundaliya, Brijesh, Hadia, S. K., "Enhancing network lifetime with an improved MOD-LEACH," International Journal of Electrical and Computer Engineering (IJECE), vol. 9, no 5, pp. 3615-3622, 2019.

[4] Savitha, S., Lingareddy, S. C., et Chitnis, Sanjay, "Energy efficient clustering and routing optimization model for maximizing lifetime of wireless sensor network," International Journal of Electrical and Computer Engineering (IJECE), vol. 10, no. 5, pp. 4798-4808, 2020.

[5] Mohammed, Samar Awad, Abd Elsalam Aly, Khaled, Ghuniem, Atef Mohammed, "An Enhancement Process for Reducing Energy Consumption in Wireless Sensor Network," International Journal of Emerging Trends in Engineering Research (IJETER), vol. 8, no 6, pp. 2765-2769, 2020.

[6] Vimala M. and Rajeev Ranjan, "Energy efficient clustering using the AMHC (adoptive multi-hop clustering) technique," Int. Journal of Electrical \& Computer Engineering (IJECE), vol. 10, no. 2, pp. 1622-1631, 2020.

[7] Vehbi Çağnı Güngör and Gerhard P. Hancke, "Industrial Wireless Sensor Networks: Applications, Protocols, and Standards," CRC Press, vol. 83, pp. 1027-1040, 2013.

[8] Aziz Mahboub, Mounir Arioua, EL Mokhtar En- naimi and Imad Ezzazi, "Performance Evolution of EnergyEfficient Clustering Algorithmsin Wireless Sensor Network," Journal of Theoretical and Applied Information Technology (JATIT), vol. 83, no. 2, pp. 187-194, 2016.

[9] W. H. Heinzelman, A. Chandrakasan and H. Balakrisham, "Energy-efficient communication protocol for wireless microsensor networks," Proc. of the 33rd Annual Hawaii Int. Conf. on System Sciences, Maui, HI, USA, 2000.

[10] Aziz Mahboub, Mounir Arioua, EL Mokhtar En-Naimi and Imad Ezzazi, "Multi-zonal approach for clustered wireless sensor networks," Int. Conf. on Electrical and Information Technologies (ICEIT), 2016, pp. 219-224.

[11] Pradeep, J. P. Mahes Kumar, Mummoorthy, "Distributed Entropy Energy-Efficient Clustering Algorithm For Heterogeneous Wireless Sensor Network Based Chaotic Firefly Algorithm Cluster Head Selection," Journal of Critical Reviews, vol. 7, no 8, pp. 1208-1215, 2020.

[12] Nehra, Vibha, Sharma, Ajay K., Tripathi, Rajiv K., "I-deec: improved deec for blanket coverage in heterogeneous wireless sensor networks," Journal of Ambient Intelligence and Humanized Computing, vol. 11, no 2, pp. 3687-3698, 2020.

[13] Syed Umar, Yerragudipadu Subbarayudu, K. Kiran Kumar and N. Bashwanth, "Designing of Dynamic Re-clustering Leach Protocol for Calculating Total Residual Time and Performance," International Journal of Electrical and Computer Engineering (IJECE), vol. 7, no. 3, pp. 1286-1292, 2017.

[14] I. F. Akyildiz, “A Survey on Sensor Networks,” IEEE Commu. Magazine, vol. 40, no. 8, pp. 102-114, 2002.

[15] EFFAH, Emmanuel THIARE, Ousmane, "Realistic Cluster-Based Energy-Efficient and Fault-Tolerant (RCEEFT) Routing Protocol for Wireless Sensor Networks (WSNs)," Future of Information and Communication Conference, vol. 1129, pp. 320-337, 2020.

[16] T. Velmurugan, "Performance based analysis between k-Means and Fuzzy C-Means clustering algorithms for connection oriented telecommunication data," Applied Soft Computing, vol. 19, pp. 134-146, 2014

[17] Radhika, M. Sivakumar, P., "Energy optimized micro genetic algorithm based LEACH protocol for WSN," Wireless Networks, vol. 27, pp. 27-40, 2020

[18] Jayarajan, P., Kanagachidambaresan, G. R., Sundararajan, T. V. P. et al., "An energy-aware buffer management (EABM) routing protocol for WSN," The Journal of Supercomputing, vol. 76, no 6, pp. 4543-4555, 2020.

[19] K. Kapitanova, S. H. Son, K. -D. Kang, "Using fuzzy logic for robust event detection in wireless sensor networks," Ad Hoc Networks, vol. 10, no. 4, pp. 709-722, 2012.

[20] Georgios Smaragdakis, Ibrahim Matta and Azer Bestavros, "SEP: A Stable Election Protocol for clustered heterogeneous wireless sensor networks," Technical Report BUCS-TR-2004-022, 2004.

[21] S. D. Muruganathan, D. C. Ma, R. I. Bhasin, and A. O. Fapojuwo, "A centralized energy-efficient routing protocol for wireless sensor networks," IEEE Communications Magazine, vol. 43, no. 3, pp. S8-13, 2005.

[22] Li Qing, Qingxin Zhu, Mingwen Wang, "Design of a distributed energy-efficient clustering algorithm for heterogeneous wireless sensor networks," Computer Communications, vol. 29, no. 12, pp. 2230-2237, 2006.

[23] P. Tyagi, R. P. Gupta, R. K. Gill, "Comparative Analysis of Cluster Based Routing Protocols used in Heterogeneous Wireless Sensor Network," Int. J. of Soft Computing and Engineering (IJSCE), vol. 1, no. 5, pp. 362-366, 2011. 
[24] D. Goyalet and M. R. Tripathy, "Routing Protocols in Wireless Sensor Networks," A Survey, pp. 474 480, 2012.

[25] Aziz Mahboub, MounirArioua, EL Mokhtar En-naimi, Imad Ezzazi and Ahmed El Oualkad, "Multi-zonal approach Clustering based on Stable Election Protocol in Heterogeneous Wireless Sensor Networks," 2016 4th IEEE International Colloquium on Information Science and Technology (CiSt), Tangier, Morocco, 2016, pp. 912-917.

[26] Aziz Mahboub, En-Naimi El Mokhtar, Mounir Arioua, Hamid Barkouk, Younes el Assari, Ahmed El Oualkadi, "An energy-efficient clustering protocol using fuzzy logic and network segmentation for heterogeneous WSN," International Journal of Electrical and Computer Engineering (IJECE), vol. 9, no 5, pp. 4192-4203, 2019.

[27] S. Singh, A. Malik, R. Kumar, "Energy efficient heterogeneous DEEC protocol for enhancing lifetime in WSNs," Engineering Science and Technology an International Journal, vol. 20, no. 1, pp. 345-353, 2017.

[28] Aziz Mahboub, MounirArioua, EL Mokhtar En- naimi and Hamid barkouk, "Distributed energy efficient clustering algorithm based on fuzzy logic approach applied for heterogeneous WSN," Proceedings of the 2nd International Conference on Computing and Wireless Communication Systems (ICCWCS 2017), pp. 1-7, 2017.

[29] Q. -T. Lam, M. -F. Horng, T. -T. Nguyen, J. -N. Lin, and J. -P. Hsu, "A High Energy Efficiency Approach Based on Fuzzy Clustering Topology for Long Lifetime in Wireless Sensor Networks," Advanced Methods for Computational Collective Intelligence, vol. 457, pp. 367-376, 2013.

[30] S. Chattopadhyay, D. K. Pratihar, and S. C. De Sarkar, "A comparative study of fuzzy c-means algorithm and entropybased fuzzy clustering algorithms," Computing and Informatics, vol. 30, no. 4, pp. 701-720, 2011.

[31] H. Wang, Z. Xu, and W. Pedrycz, "An overview on the roles of fuzzy set techniques in big data processing: Trends, challenges, and opportunities," Knowledge-Based Systems, vol. 118, pp. 15-30, 2017.

[32] Aziz Mahboub, MounirArioua, EL Mokhtar En- naimi and Hamid Barkouk, "Fuzzy C-Means based Hierarchical Routing approach for homogenous WSN," Proceedings of the Mediterranean Symposium on Smart City Applications, vol. 37, pp. 265-275, 2017.

[33] Ali Abdul-hussian Hassan, Wahidah Md Shah, Mohd Fairuz Iskandar Othman and Hayder Abdul Hussien Hassan, "Evaluate the performance of K-Means and the fuzzy C-Means algorithms to formation balanced clusters in wireless sensor networks," International Journal of Electrical \& Computer Engineering (IJECE), vol. 10, no. 2, pp. 1515-1523, 2020.

\section{BIOGRAPHIES OF AUTHORS}

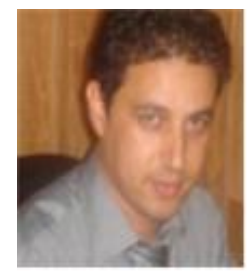

Hamid Barkouk He obtained Master Degree in Computer Sciences from Faculty of Sciences, University of Abdelmalek Essaâdi in 2015, he is currently a $\mathrm{PhD}$ Student in LIST (Laboratoired'InformatiqueSystemes et Telecommunications), Department of Computer Sciences, Faculty of Sciences and Technologies, Tangier, Morocco at the University of Abdelmalek Essaadi. His research interests include wireless sensor networks, wireless networking and communication. He is an Author/Co-Authors of several Articles and Chapters, published in The International Journals and in the conference proceedings as well as Chapters of books, in Computer Sciences.

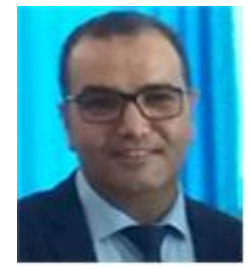

El Mokhtar En-Naimi is a Full Professor in the University of Abdelmalek Essaâdi, Faculty of Sciences and Technologies of Tangier, Department of Computer Sciences. (He was Temporary Professor: from 2000 to 2003 and Professor Permanent: since 2003/2004 and actually, He is a Full Professor). He is a Head of Computer Sciences Department, since October 2016 until now. He was responsible for a Bachelor of Science and Technology, BST Computer Engineering ("Licence LST-GI"), from January 2012 to October 2016. He is also a founding member of the Laboratory LIST (Laboratoire d'Informatique, Systèmes et Télécommunications), the University of Abdelmalek Essaâdi, FST of Tangier, Morocco. He is also an Expert Evaluator with the ANEAQ, since the academic year 2016/2017 until now, that an Expert of the Private Establishments belonging to the territory of the UAE and also an Expert of the Initial or Fundamental Formations and Formations Continuous at the Ministry of Higher Education, Scientific Research and Executive Training and also at the UAE University and the FST Tangier since 2012/2013 until Now. He is an Author/Co-Authors of several Articles, published in The International Journals in Computer Sciences, in particular, in Multi-Agent Systems (MAS), Cases Based Reasoning (CBR), Artificial Intelligent (AI), eLearning, MOOC, Big DATA, Data-mining, Wireless Sensor Network, VANet, MANet, and Smart City. He is also Director of several Doctoral Theses in Computer Sciences. In addition, he is an associate member of the ISCN - Institute of Complex Systems in Normandy, the University of the Havre, France, since 2009 until Now.

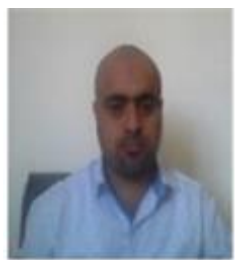

Aziz Mahboubis a Professor in the University of Abdelmalek Essaâdi, Faculty of Sciences and Technologies of Tangier, Department of Computer Sciences, since September 2019 until Now. He is a member ofthe Laboratory LIST (Laboratoire d'Informatique, Systèmes et Télécommunications), the University of Abdelmalek Essaâdi, FST of Tangier, Morocco. He obtained Master Degree in Computer Sciences from Faculty of Sciences, University of Abdelmalek Essaâdi in 2008. He obtained His Doctorate (PhD) in Computer Sciences from Faculty of Sciences and Technologies of Tangier, Department of Computer Sciences, University of Abdelmalek Essaâdi in April 2019. His researches are in fields of Wireless Sensor Network, Internet of Things, wireless communications and mobile computing. He has served as invited reviewer. He has published research papers in IJECE Journal, JATIT Journal, and conference proceedings as well as chapters of books. 